\title{
Puentes de Miocardio en Corazones de Avestruz (Struthio Camelus)
}

\author{
Miocardial Bridge in Ostrich Heart (Struthio Camelus)
}

Consuelo Troncoso ${ }^{* * * *}$ \& Jorge Henríquez Pino ${ }^{* * *}$

TRONCOSO, C. \& HENRÍQUEZ, J. P. Puentes de miocardio en corazones de avestruz (Struthio camelus). Int. J. Morphol., 33(2):666$672,2015$.

RESUMEN: El término puente de miocardio es atribuido al conjunto de fibras musculares cardiacas, que a veces se sobreponen a un segmento subepicárdico de una determinada rama de las arterias coronarias derecha e izquierda. Polacek (1959) fue el primero en enfocar esta entidad desde el punto de vista muscular, de aquí nace el nombre de "puente de miocardio" siendo actualmente la más usada en clínica. La presencia de estos puentes, ha sido identificada tanto en humanos como en animales y basados en ello, se estudiaron 50 corazones de avestruz, de la clase African Black, cuyo objetivo fue determinar presencia de puentes de miocardio, número, longitud y principales arterias en donde se ubican. Hubo presencia de puentes de miocardio en $20(40,0 \%)$ de los 50 corazones estudiados; el número total de puentes de miocardio fue de 34 , de los cuales $30(88,2 \%)$ se ubicaron sobre la arteria coronaria izquierda y sus ramas y $4(11,8 \%)$ sobre la arteria coronaria derecha y sus ramas. El número de puentes varió de 1 a 4 por corazón. Los vasos donde se observaron con mayor frecuencia fueron: ramas ventriculares de la rama interventricular paraconal con $22(64,7 \%)$ puentes y rama interventricular paraconal con $8(23,5 \%)$ puentes. Los registros métricos de la extensión de los puentes de miocardio variaron entre 1,6 mm y 73,1 mm.

PALABRAS CLAVE: Corazón; Puentes de miocardio; Avestruz.

\section{INTRODUCCIÓN}

El término puente de miocardio es atribuido al conjunto de fibras musculares cardíacas, que a veces se sobreponen a un segmento subepicárdico de una determinada rama de las arterias coronorias derecha o izquierda (Bezerra, 1982; Amaral, 1989; Severino \& Bombonato, 1992).

Cuando un segmento de una arteria coronaria principal cursa a través del miocardio se la considera como puente de miocardio (Polacek, 1959); también se ha descrito como una banda de tejido muscular que se encuentra esporádicamente sobre la arteria coronaria, en los seres humanos (Morales et al., 1980) y en ciertos animales como suinos (Bertolini et al., 1994), perros (Amaral \& Bombonato, 1996), gatos (Bombonato et al., 1991), león de montaña (Santos et al., 2004), bovinos azebuados (Severino et al., 1997), entre otros.

Esta disposición muscular fue inicialmente descrita por Crainicianu (1922) aunque ya se le reconocía desde hace más de doscientos años. Polacek es el primero en enfocar esta característica desde el punto de vista muscular, de aquí nace el nombre de "puente de miocardio" que realmente re- vela una disposición anatómica especial en el corazón, siendo actualmente la más usada en clínica.

Décourt et al. (1980) analizaron sobre si el puente de miocardio es una anomalía o debe ser visto como una variación anatómica. Basándonos en la literatura consultada se observa la carencia de trabajos que describan los aspectos relacionados al conocimiento de la morfología y funcionalidad de los puentes de miocardio en aves. Por esta razón nuestro estudio pretende contribuir al conocimiento de la frecuencia, longitud y localización de puentes de miocardio sobre las ramas ventriculares de las arterias coronarias derecha e izquierda en corazón de avestruz.

\section{MATERIAL Y MÉTODO}

En el estudio fueron utilizados 50 corazones de avestruz, de la clase African Black sin aparente patología cardiaca, cuyas edades fluctuaron entre los 12 y 15 meses, sin especificación de sexo, con un peso que varió entre 370,5

\footnotetext{
* Programa de Magister en Anatomía, Universidad de La Frontera, Temuco, Chile.

** Disciplina de Anatomía Humana, Universidad Santo Tomás, Sede, Temuco, Chile.

**** Departamento de Ciencias Básicas, Facultad de Medicina, Universidad de La Frontera, Temuco, Chile.
} 
g y $1050 \mathrm{~g}$ y provenientes del criadero El Carmen de Calera de Tango, Región Metropolitana, Chile.

Mediante masajes manuales sucesivos, se vaciaron atrios y ventrículos, posteriormente fueron lavados con agua corriente. Las muestras obtenidas fueron identificadas mediante el uso de placas plásticas numeradas y los datos correspondientes a cada una se anotaron en un registro ad-hoc.

Luego, las arterias coronarias fueron inyectadas a través de sus ostios, con Látex Neopreno 650, Härting S.A. Industrias Químicas, tiñendo de colorante amarillo la arteria coronaria derecha y con rojo la arteria coronaria izquierda. Posteriormente los corazones fueron inmersos en solución acuosa de formol al 10\% durante un periodo de 10 a 12 días.

Se disecaron las arterias coronarias y sus ramas con la ayuda de instrumental quirúrgico convencional y lupa estereoscópica.

Finalizada la disección se recolectaron los datos relativos a la frecuencia, longitud y localización de los puentes de miocardio sobre las ramas de las arterias coronarias derecha e izquierda. Con la ayuda de un Caliper digital, se tomaron las medidas relativas a la longitud de los mismos. Se realizaron esquemas sobre las arterias y sus ramas con los respectivos puentes y se fotografiaron las muestras más representativas.

\section{RESULTADOS}

Los resultados obtenidos de la presencia de los puentes se muestran en la Tabla I (Figs. 1 y 2).

La distribución de los puentes de miocardio en relación a las arterias coronarias y sus ramas se muestran en la Tabla II. (Figs. 3 y 4).

Tabla I. Número de puentes de miocardio.

\begin{tabular}{lcc}
\hline \multirow{2}{*}{ Número de Puentes } & \multicolumn{2}{c}{ Corazones } \\
\cline { 2 - 3 } & n & \% \\
\hline 1 & 11 & 55 \\
2 & 5 & 25 \\
3 & 3 & 15 \\
4 & 1 & 5 \\
Total & 20 & 100 \\
\hline
\end{tabular}

Tabla II. Distribución de puentes de miocardio según ramas arteriales.

\begin{tabular}{lcc}
\hline \multirow{2}{*}{ Vasos arteriales } & \multicolumn{2}{c}{ Puentes de miocardio } \\
\cline { 2 - 3 } & $\mathbf{n}$ & $\mathbf{\%}$ \\
\hline R. Paraconal & 8 & 23,53 \\
R. Lateral & 22 & 64,70 \\
R. Subsinuosa & 3 & 8,83 \\
R. Colateral & 1 & 2,94 \\
Total & 34 & 100 \\
\hline
\end{tabular}

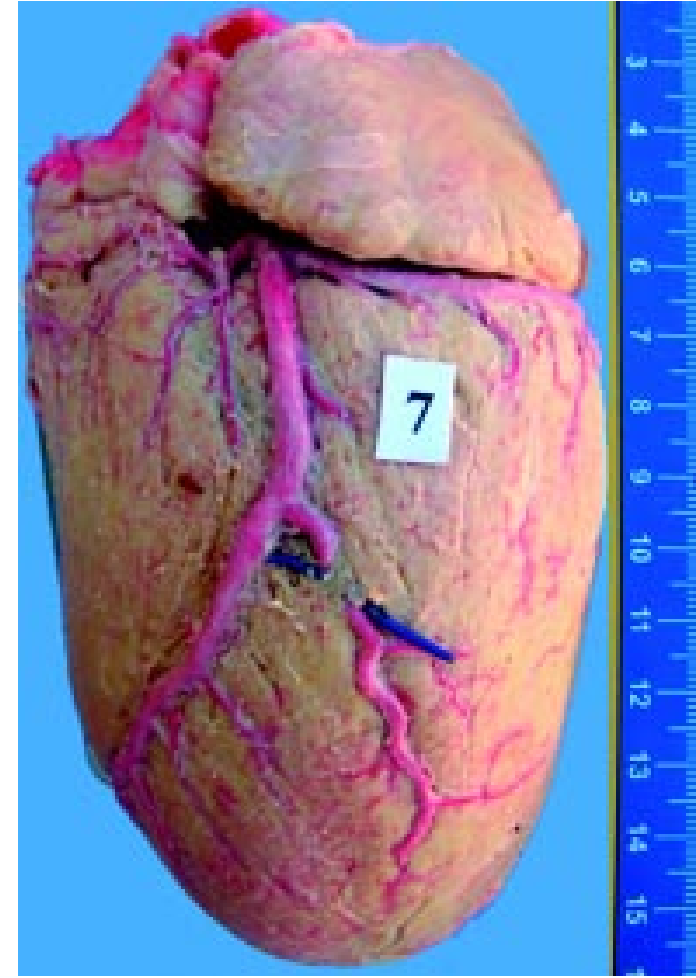

Fig. 1. Observación $\mathrm{N}^{\mathrm{o}}$ 7. Se observa un (1) puente de miocardio de $6,30 \mathrm{~mm}$, sobre una rama ventricular colateral de la rama interventricular paraconal.

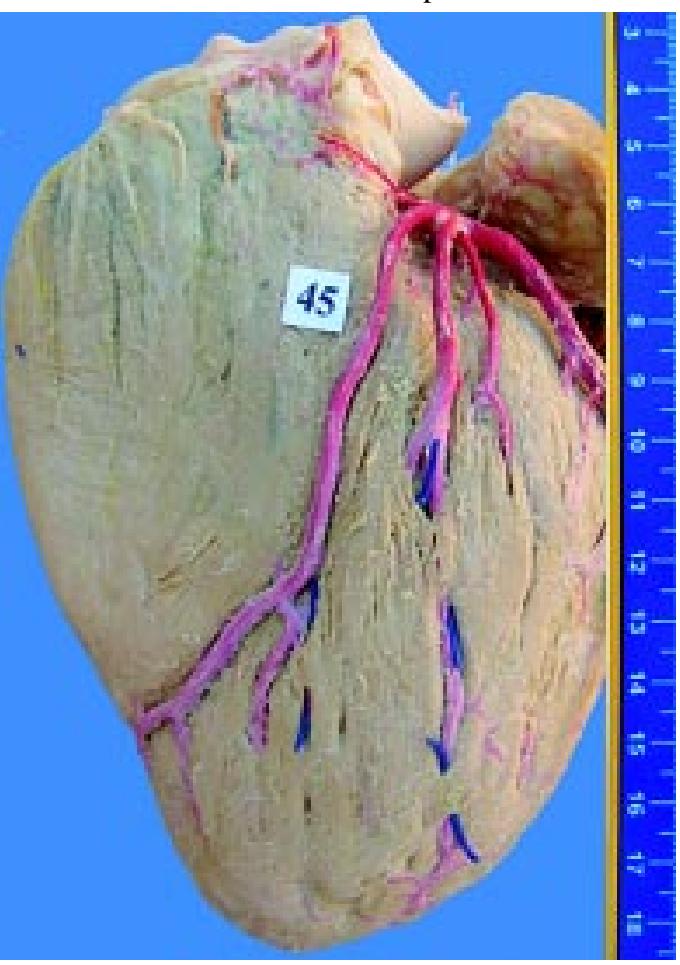

Fig. 2. Observación $\mathrm{N}^{\mathrm{o}} 45$. Se observan tres (3) puentes de miocardio, uno sobre la rama ventricular colateral de la Rama Interventricular Paraconal y dos sobre una rama ventricular de la rama circunfleja. 


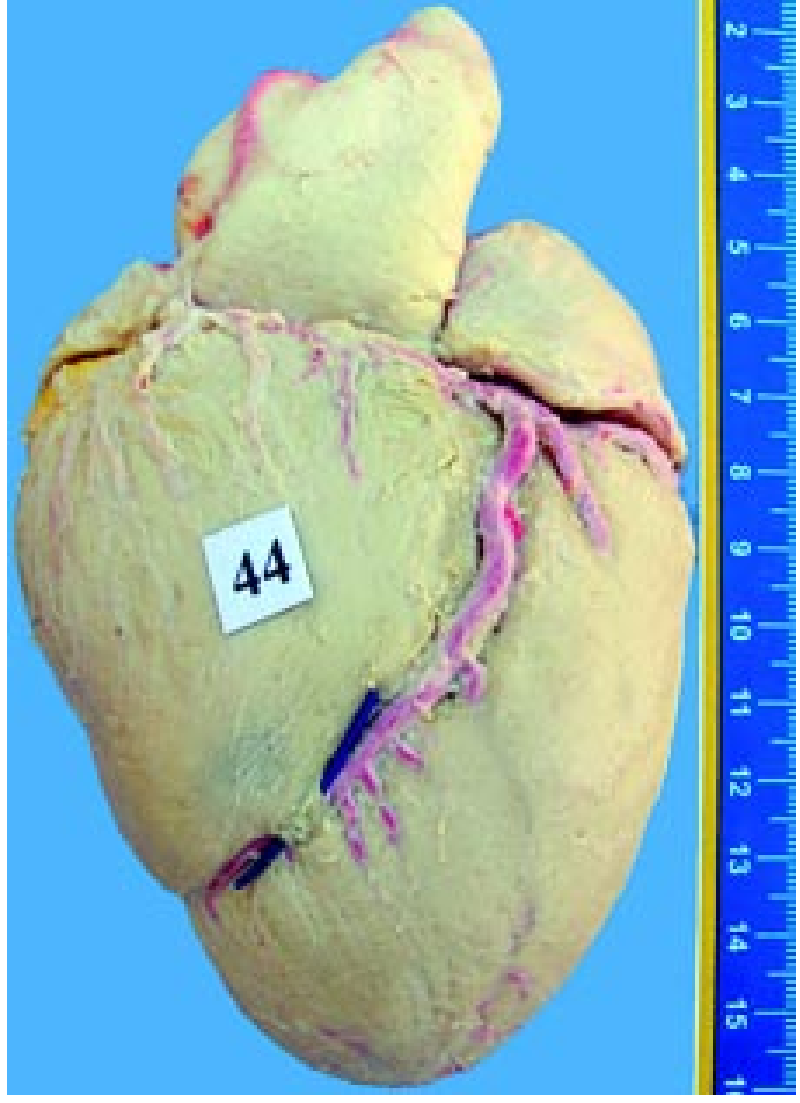

Fig. 3. Observación $\mathrm{N}^{\circ}$ 44. Se observa un puente de miocardio de $9,73 \mathrm{~mm}$, directamente sobre la rama interventricular paraconal de la arteria coronaria izquierda.

Tabla III. Longitud de los puentes de miocardio.

\begin{tabular}{lcc}
\hline Longitud (mm) & n & \% \\
\hline $1,0-10,0$ & 24 & 70,59 \\
$10,1-20,0$ & 6 & 17,64 \\
$20,1-30,0$ & 2 & 5,89 \\
$30,1-40,0$ & 1 & 2,94 \\
$40,1-70,0$ & 0 & --- \\
$70,1-80,0$ & 1 & 2,94 \\
Total & 34 & 100,0 \\
\hline
\end{tabular}

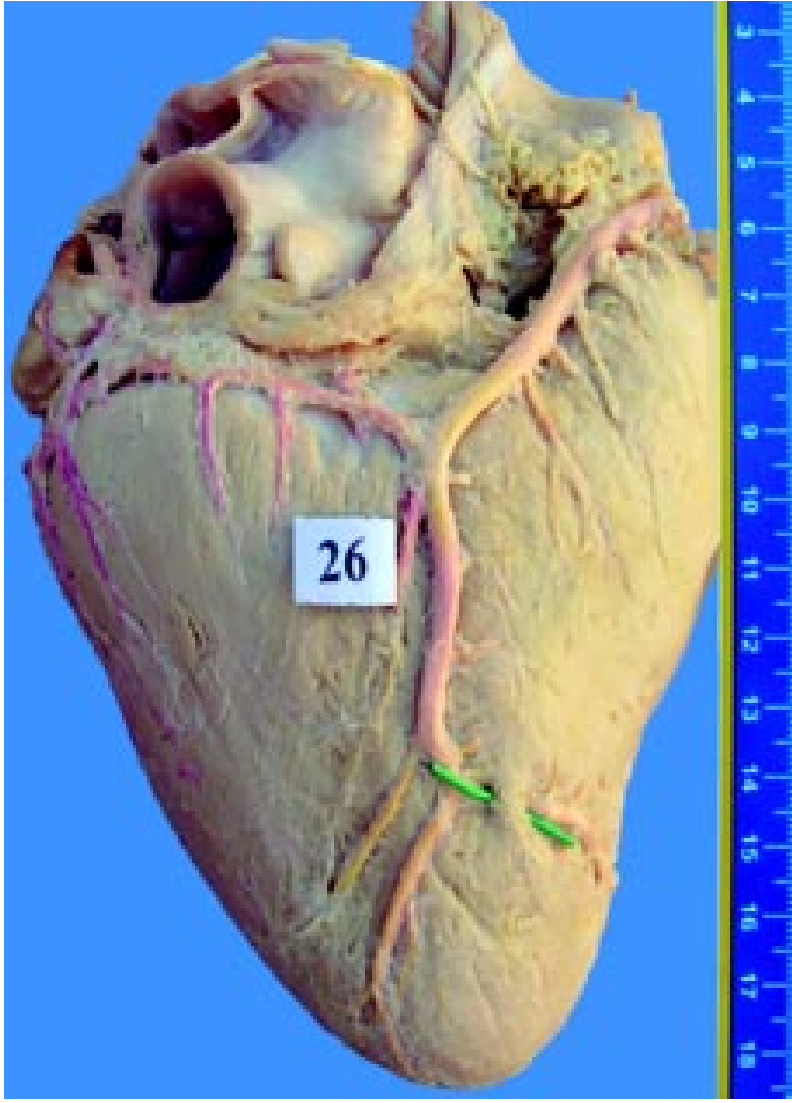

Fig. 4. Observación $\mathrm{N}^{\circ}$ 26. Se observa un puente de miocardio de $8,89 \mathrm{~mm}$ sobre una rama ventricular colateral de la rama interventricular subsinuosa, de la arteria coronaria derecha.

La longitud promedio de los puentes de miocardio esta expresada en la Tabla III (Fig. 5). La longitud promedio de los puentes de miocardio según las arterias coronaria derecha e izquierda y sus ramas se observa en la Tabla IV. La longitud promedio de los puentes de miocardio, respecto a las ramas que con mayor frecuencia los presentan se observa en la Tabla V.

Tabla IV. Longitud promedio de los puentes de miocardio sobre las ramas de las arterias coronarias derecha e izquierda.

\begin{tabular}{lcccc}
\hline \multicolumn{1}{c}{ Arterias } & $\mathbf{n}$ & $\begin{array}{c}\text { Límites } \\
(\mathbf{m m})\end{array}$ & $\begin{array}{c}\text { Promedio } \\
(\mathbf{m m})\end{array}$ & DE \\
\hline Coronaria derecha & 4 & $1,62-8,89$ & 4,35 & 3,31 \\
Coronaria izquierda & 30 & $2,49-73,1$ & 12,35 & 13,92 \\
\hline
\end{tabular}

Tabla V. Longitud promedio de los puentes de miocardio respecto a los ramas arteriales de mayor frecuencia.

\begin{tabular}{lcccc}
\hline Ramas arteriales & $\mathbf{n}$ & $\begin{array}{c}\text { Límites } \\
(\mathbf{m m})\end{array}$ & $\begin{array}{c}\text { Promedio } \\
(\mathbf{m m})\end{array}$ & DE \\
\hline Paraconal & 8 & $4,09-73,1$ & 15,31 & 23,46 \\
Lateral & 22 & $2,49-39,52$ & 11,27 & 8,93 \\
\hline
\end{tabular}




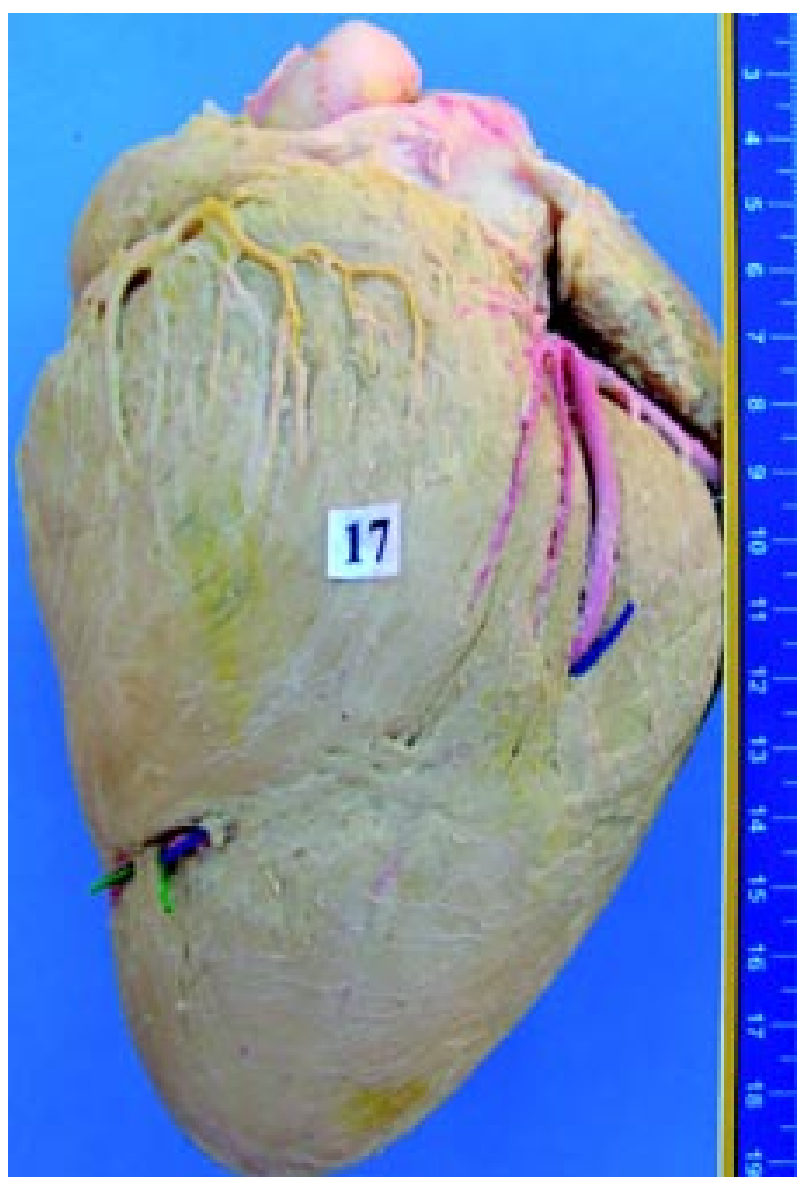

Fig. 5. Observación $\mathrm{N}^{\circ}$ 17. Se observan dos puentes de miocardio sobre la rama interventricular paraconal, el proximal es de 73,1 $\mathrm{mm}$ y el distal de $1,83 \mathrm{~mm}$.

\section{DISCUSIÓN}

Las arterias coronarias y sus ramas principales están generalmente ubicadas bajo el pericardio. Sin embargo algunos individuos poseen regiones en que el músculo cardiáco cubre estos vasos, en forma de puente, los que pueden ser vistos después de remover el epicardio y el tejido subepicárdico.

Los puentes de miocardio en la literatura anatómica han recibido diferentes denominaciones para poder identificarlos, entre las cuales podemos citar: "arteria sumergida" (Tandler, 1913), "arteria coronaria mural" (Geiringer, 1951), "vaso intramural" (Edwards et al., 1956), "arteria intraparietal" (Penther et al., 1977), "arteria coronaria intramural" (Marshall \& Headley, 1978). Sin embargo Polaceck fue el primero en enfocar esta característica desde el punto de vista muscular, denominándole puente muscular, de aquí nace el término "puente de miocardio" que es la acepción más aceptada en la terminología médico-biológica.
Muchos autores relacionan los puentes de miocardio con diversas patologías cardíacas. Hay que hacer notar que Berg (1963) al estudiar esta estructura en corazones de suinos la clasificó como una "anomalía", término que no compartimos pues la consideramos como una variación anatómica.

Debido a la escasez de datos en la literatura veterinaria y aviar referente a los aspectos morfológicos del avestruz, y en especial sobre puentes de miocardio, esta investigación ha tenido por objetivo ofrecer datos que contribuyan a ampliar el conocimiento de los puentes de miocardio en esta especie y al mismo tiempo es pertinente señalar la poca información que existe sobre estudios de puentes de miocardio en aves, por lo cual esta discusión se basará en investigaciones realizadas en diversas especies animales.

En nuestra serie se pudo constatar que en el 40,0\% de ellos se evidenciaban puentes de miocardio, resultado similar al obtenido por Bombonato et al. (1994), en estudios realizados en suinos con un $36,4 \%$ y Amaral \& Bombonato en perros con un $45,5 \%$, respectivamente.

Otros investigadores como Machado et al. (1988) observaron estos puentes en pecarí en un 78,6\%; Bombonato et al. (1991) en gatos en un 66,7\%; Teofilovski et al. (1992) en monos en un 82,7\%; Machado et al. (1995) en caprinos en un 94,6\%; Nocetti et al. (2002) en jabalí en un 57,1\% y Ribeiro et al. (2009) en mulas en un $76,6 \%$.

Trabajos realizados en bovinos azebuados (Severino et al., 1997), bovinos raza canchin (Santos et al., 2000), ovinos raza Ideal (Cruz et al., 2007) registran una frecuencia de $100 \%$ de corazones con presencia de puentes de miocardio.

El número total de puentes de miocardio encontrados en el corazón de avestruz fue de 34 puentes, similar al obtenido en monos (Teofilovski et al.) ( $\mathrm{n}=33$ ), suinos (Bombonato et al., 1994) y gatos (Bombonato et al., 1991). Otros estudios han indicado un número menor, Nocetti et al., en jabalí con 26 puentes y Cruz et al., en ovinos con 20 puentes. Otros han señalado un número mayor como Ribeiro et al., en mulas con 64 puentes; Machado et al. (1995) en caprinos con 64 puentes, y Amaral \& Bombonato en perros con 94 puentes, encontrándose cifras muy elevadas en bovinos Azebuados (Severino et al.) con 106 puentes y bovinos raza Canchin (Santos et al., 2000) con 337 puentes.

En lo que se refiere a la presencia de puentes de miocardio en un mismo corazón, en avestruz el número varió de 1 a 4 puentes, cifra semejante a la encontrada por Machado et al. (1995) en caprinos, mientras que según Bombonato et al. $(1991,1994)$ y Teofilovski et al., en en ga- 
tos, suinos y monos, varían entre 1 y 3 puentes, respectivamente.

En otras especies, como perros se encontraron de 1 a 5 (Amaral \& Bombonato); en bovinos Azebuados (Severino et al.) y en mulas Ribeiro et al. de 1 a 7 puentes, mientras que Santos et al. (2000), en bovinos raza Canchin su número varío de 5 a 26 puentes. Por su parte Cruz et al., en ovinos, encontró un puente por corazón y Machado et al. (2002) no evidenció presencia de puentes de miocardio en ciervo de los pantanos.

Un caso reportado en león de montaña indicó la presencia de 12 puentes de miocardio (Santos et al., 2004). Bezerra et al. (1985) examinando un corazón de camello describió la presencia de 6 puentes de miocardio.

En cuanto a la longitud de los puentes de miocardio en corazones de avestruz ésta varió de 1,6 $\mathrm{mm}$ a 56,5 $\mathrm{mm}$ con un promedio de $10,5 \mathrm{~mm}$, cifra similar a la encontrada en mulas por Ribeiro et al., con un promedio de longitud de 9,6 $\mathrm{mm}$ y en perros que se observó una longitud promedio de $8,9 \mathrm{~mm}$ (Amaral \& Bombonato).

Estudios realizados en otras especies mostraron una longitud promedio inferior a la encontrada en el presente estudio, como es el caso de Machado et al. (1988), en pecarí, con una longitud promedio de 4,5 $\mathrm{mm}$ y trabajos realizados por Bombonato et al. (1994), en suinos con 7,5 mm y Bombonato et al. (1991), en gatos con 3,4 mm.

Resultados mayores fueron encontrados por otros investigadores Santos et al., en bovinos raza Canchin con 12,4 $\mathrm{mm}$, Severino \& Bombonato en bovinos Gir, Guzera Indubrasil con 13,3 mm; Nocetti et al., en jabalí con 14,1 mm; Severino et al. en bovinos Azebuados con 16,2 mm; Machado et al. (1995), en caprinos con $22.0 \mathrm{~mm}$ y Cruz et al., en ovinos raza Ideal con $22,8 \mathrm{~mm}$.

En relación a la localización de los puentes de miocardio en las arterias coronarias pudimos constatar que la mayor parte de los puentes de miocardio se identificaron en las ramas de la arteria coronaria izquierda con un $88,2 \%$, resultado similar al observado en estudios realizados por Santos et al. (2000) en bovinos raza Canchin con un 86,9\%; Teofilovski et al., en monos con un 90,0\% y Amaral \& Bombonato en perros con un $90,4 \%$.

Cifras superiores a las encontradas por nosotros nos entregan los trabajos realizados por Severino et al. en bovinos Azebuados con un 94,3\%; Cruz et al., en ovinos raza Ideal con un $96,0 \%$ de puentes en las ramas ventriculares de la arteria coronaria izquierda, mientras que en otros estudios anató- micos realizados indican cifras muy diferentes a las nuestras como es el caso de los gatos Bombonato et al. (1991), con $56,7 \%$ ) y en caprinos con un $100 \%$ de puentes en la arteria coronaria izquierda Machado et al. (1995); Ribeiro et al., en mulas pudieron constatar igual frecuencia de puentes de miocardio sobre ramas de la arteria coronaria derecha e izquierda con un $37,5 \%$.

En el caso del león de montaña se evidenciaron los puentes de miocardio en un $100 \%$ en las ramas interventricular de la arteria coronaria izquierda (Santos et al., 2004).

La presencia de puentes de miocardio con mayor frecuencia en ramas de la arteria coronaria derecha, fue observada en pecarí en donde Machado et al. (1988), evidenciaron puentes de miocardio en un 53,6\%, resultado similar al observado por Nocetti et al., en jabalí con un 53,8\%, mientras que Amaral \& Bombonato en perros, encontraron que un $65,6 \%$ de los corazones presentaban puentes de miocardio en esta arteria.

De los 34 puentes de miocardio en corazón de avestruz, el $64,7 \%$ se evidenció en las ramas ventriculares ventrales de la rama interventricular paraconal de la arteria coronaria izquierda, mientras que el $23,5 \%$ se encontró directamente sobre la rama interventricular paraconal. Una menor incidencia fue observada directamente sobre la rama interventricular subsinuosa $(8,8 \%)$ y en ramas ventriculares dorsales de la interventricular subsinuosa $(2,9 \%)$.

Estudios realizados en diversas especies evidenciaron que los puentes de miocardio se localizaban con mayor frecuencia en la rama interventricular paraconal, tal es el caso de Cruz et al., que observaron en ovinos raza Ideal un 96,0\%, Amaral \& Bombonato en perros un 65,9\%, Machado et al. (1995) en caprinos un 54,7\%, y Bombonato et al. (1991) en gatos un $50,0 \%$.

Otros trabajos entregan información divergente a la nuestra en donde la localización de los puentes de miocardio es mayoritariamente en la rama interventricular subsinuosa de la arteria coronaria derecha, tal es el caso de Machado et al. (1988) en pecarí con un 50,0\%, Bombonato et al. (1994) en suinos con un 46,9\% y Nocetti et al., en jabalí con un 42,3\%.

Considerando que la mayoría de las aves tienen sus arterias coronarias localizadas en la pared del corazón, es decir son intramurales, en el avestruz podemos considerar que las características de su corazón, en este aspecto, es de animal mamífero, no existe literatura en relación al tema en avestruz, por lo cual tuvimos que comparar nuestro estudio con animales mamíferos, por lo que esperamos que esta investigación sea un aporte a un tema no estudiado anteriormente. 
TRONCOSO, C. \& HENRÍQUEZ, J. P. Miocardial bridge in ostrich heart (Struthio camelus). Int. J. Morphol., 33(2):666-672, 2015.

SUMMARY: The term myocardial bridge is attributed to all cardiac muscle fibers, which sometimes overlap a subepicardial segment of a particular branch of the right and left coronary arteries. Polacek in 1959 was the first to approach this entity from the standpoint of muscle. From this arises the name of "myocardial bridge" and is currently the term most widely used clinically. The presence of these bridges, has been identified in humans and in animals and based on that, 50 African Black class ostrich hearts were studied, with the aim to determine the presence of myocardial bridges, number, length and main arteries where they are located. There was presence of myocardial bridges in $20(40.0 \%)$ of the 50 hearts studied, the total number of myocardial bridges was 34 , of which 30 $(88.2 \%)$ were located on the left coronary artery and its branches and $4(11.8 \%)$ over the right coronary artery and its branches. The number of bridges ranged from 1 to 4 by heart. The vessels most frequently observed were: ventricular branches paraconal interventricular branch with $22(64.7 \%)$ and interventricular branch paraconal bridges with $8(23.5 \%)$ bridges. Metrical records of the extent of myocardial bridges ranged between $1.6 \mathrm{~mm}$ and $73.1 \mathrm{~mm}$.

KEY WORDS: Heart; Myocardial bridge; Ostrich.

\section{REFERENCIAS BIBLIOGRÁFICAS}

Amaral, R. C. Pontes de miocardio em cães. Tesis de Doctorado. Sao Pãulo, Facultad de Medicina Veterinaria y Zootecnia de la Universidad de São Paulo, 1989.

Amaral, R. C. \& Bombonato, P. P. Ponte de miocárdio em caes: I. Frequencia e largura. Braz. J. Vet. Res. Anim. Sci., 33(3):1539, 1996.

Berg, R. Über das Auftreten von Myocardbrücken Über den Koronargefässen beim Schwein (Sus scrofa domesticus). Anat. Anz., 112:25-31. 1963.

Bertolini, S. M. M. G.; Prates, N. E. V. B. \& Neto, M. H. M. Estudo macroscópico estatístico das pontes de miocárdio sobre as artérias coronárias de suinos. Rev. Unimar, 16(2):383-93,1994.

Bezerra, A. J.; DiDio, A. S. \& DiDio, L. J. Bridges of myocardium over branches of the coronary arteries in Camelus dromedarius. Arch. Ital. Anat. Embriol., 90(4):267-74, 1985.

Bezerra, A. J. Contribuição para o conhecimento das pontes de miocárdio. Tesis de Doctorado. Sao Pãulo, Escuela Paulista de Medicina, 1982.

Bombonato, P. P.; Domingos, C. O., Mariana, A. N. B.; Silva, F. O. C. \& Intelizano, W. Ocorrência de pontes de miocárdio em suínos. Braz. J. Vet. Res. Anim. Sci., 31(2):107-11, 1994.

Bombonato, P. P.; Amaral, R. C.; Mariana, A. N. B.; Hokamura, H. K.; Santos, A. L.; Silva, F. O. C. \& Severino, R. S. Pontes de miocárdio em gatos. Rev. Cent. Ciênc. Bioméd. Univ. Fed. Uberlândia, 7(1):49-57, 1991.

Crainicianu, A. Anatomische Studien über die Koronararterien und Experimentelle Untersuchungen über ihre Durchgangigkeit. Virchows. Arch. Path. Anat. Physiol., 238:1-75, 1922.

Cruz, T. L.; Marcal, A. V.; Bombonato, P. P.; Benedicto, H. G.; Silva, F. O. C.; Severino, R. S.; Smrreaux, P. G. \& Blasquez,
F. J. H. Pontes de miocárdio em ovinos da raça Ideal: frecüência e largura. Ciênc. Anim. Bras., 8(2):307-12, 2007.

Décourt, L. V.; Carvalho, V. B. \& Martinez, J. R. M. Ponte miocárdica: uma entidade controvertida. Rev. Hosp. Clin. Fac. Med. S. Paulo, 35:157-60, 1980.

Edwards, J. C.; Burnsides, C.; Swarm, R. L. \& Lansing, A. I. Arteriosclerosis in the intramural and extramural portions of coronary arteries in the human heart. Circulation, 13(2):235$41,1956$.

Geiringer, E. The mural coronary. Am. Heart J., 41(3):359-68, 1951.

Machado, M. R. F.; Borges, E. M.; Oliveira, F. S.; FilippiniTomazini, M.; Melo, A. P. F. \& Duarte, J. M. B. Intramyocardial course of the coronary arteries in the marsh deer (Blastocerus dichotomus). Braz. J. Vet. Res. Anim. Sci., 39(6):285-7, 2002.

Machado, M. R. F.; Bombonato, P. P.; Mariana, A. N. B.; Severino, R. S. \& Amaral, R. C. Pontes de miocárdio em caprinos. Ars Vet., 11(1):17-24, 1995.

Machado, G. V.; Lesnau, G. G. \& Cavalcante Filho, M. F. Ocorrência de pontes de miocárdio em Queixadas (Tayassu pecari). Arq. Ciên. Vet. Zool. UNIPAR, 1(1):59-65, 1988.

Marshall, M. E. \& Headley, R. N. Intramural coronary artery as a cause of unstable angina pectoris. South Med. J., 71(10):1304$6,1978$.

Morales, A. R.; Romanelli, R. \& Boucek, R. J. The mural left anterior descending coronary artery, strenuous exercise and sudden death. Circulation, 62(2):230-7, 1980.

Nocetti, L. M.; Bombonato, P. P.; Santana, M. I. S.; Carneiro e Silva, F. O. \& Severino, R. S. Pontes de miocárdio em corações de javali. Braz. J. Vet. Res. Anim. Sci., 39(2):66-73, 2002. 
Penther, P.; Blanc, J. J.; Boschat, J. \& Granatelli, D. Intramural anterior interventricular artery. Anatomical study. Arch. Mal. Coeur Vaiss., 70(10):1075-9, 1977.

Polacek, P. Muscular bridges and loops on coronary arteries of man. Cs. Morfologie, 7:119-25, 1959.

Ribeiro, A. L. C.; Severino, R. S.; Guerra, R. R.; Favaron, P. O.; Tommasi Junior, H. L. P.; Ricci, R. E. G.; Franciolli, A. L. R.; Facciotti, P. R. \& Bombonato, P. P. Biometria de pontes de miocárdio em muares (Equus caballus x Equus asinus Linnaeus 1758). Biotemas, 22(3):177-84, 2009.

Santos, J. W.; Bombonato, P. P.; Beletti, M. E.; Severino, R. S. \& Carneiro e Silva, F. O. Pontes de miocárdio em bovinos da raça Canchim, I - Aspectos microscópicos. Braz. J. Vet. Res. Anim. Sci., 37(2):128-31, 2000.

Santos, A. L. Q.; Carvalho, S. F. M.; Moraes, F. M. \& Alves Júnior, J. R. F. Myocardial bridges in mountain lion (Puma concolor, Jardine-1834) (Felidae): A case report. Braz. J. Morphol. Sci., 21(4):221-3, 2004.

Severino, R. S. \& Bombonato, P. P. Ocorrência de pontes de miocárdio em bovinos das raças Gir, Guzerá, Indubrasil e Nelore. Braz. J. Vet. Res. Anim. Sci., 29 (1):15-30, 1992.

Severino, R. S.; Carneiro de Silva, F. O.; Santos, A. L. Q.; Drummond, S. S.; Bombonato, P. P.; Duran, F. P. \& Marçal, A. V. Pontes de miocárdio em bovinos azebuados. Braz. J. Vet. Res. Anim. Sci., 34(5):288-91, 1997.

Tandler, J. Anatomie des Herzens. En: Bardeleben, K. (Ed.). Handbuch der Anatomie des Menschen. Jena, Gustav Fischer Verlag, 1913.

Teofilovski, G.; Filipovic, B.; Bogdanovic, D.; Trpinac, D.; Rankovic, A.; Stankovic, G. \& DiDio, L. J. Myocardial bridges over coronary arteries in Cercopithecus. Ann. Anat., 174(5):435-9, 1992.

\author{
Dirección para Correspondencia: \\ Dr. Jorge Henríquez Pino \\ Facultad de Medicina \\ Universidad de La Frontera \\ Casilla 54-D \\ Temuco \\ CHILE
}

Email: jorge.henriquez@ufrontera.cl

Recibido: 15-01-2015

Aceptado: 22-04-2015 